\title{
Spectrophotometry of the zodiacal light
}

\author{
J. F. James \\ 29, Merchants' Quay, Salford, M5 2XF, England
}

(Received October 7, 1997; Revised March 8, 1998; Accepted March 8, 1998)

\begin{abstract}
The history of zodiacal light spectrometry follows faithfully the history of optical detection technology. Each new design effort has resulted in the harnessing of new and more efficient detectors to the problem, and with each new development the quality of the spectra obtained has improved. This article traces the development of the technique from its beginnings in the 1950's to the spectrographs of the present day.
\end{abstract}

\section{Introduction}

The zodiacal light is an example of those very feeble extended sources of light which, because of their nature, have resisted spectral analysis until recent times. The advent, firstly of photomultipliers, and more recently of the cooled CCD camera has eased the problems somewhat although many remain, and the history of zodiacal light spectroscopy is essentially the history of photon-sensing technology and of optical design. The story can be divided conveniently into three parts:

- Silver-halide photography with optical spectrographs of high numerical aperture.

- Monochromators of high efficiency with photoncounting photomultiplier detectors.

- Interference spectrographs with cooled CCD cameras.

\section{Silver Halide Spectrography}

The photographic emulsion suffers notoriously from reciprocity failure. That is to say, the response to a total exposure falls at long exposures, so that the effective exposure, the light intensity $\times$ the square of the numerical aperture $\times$ the exposure time is not constant, but falls at exposure times longer than a few seconds.

There are several ways of improving the sensitivity of a spectrograph in these circumstances. For example:

- The highest numerical aperture may be used, as in a Schmidt camera.

- The photographic emulsion may treated with a reducing agent such as hydrogen or ammonia to reduce its reciprocity failure.

- The emulsion may be refrigerated.

And all these techniques may be used together.

It was with a Schmidt system that Ingham (Blackwell and Ingham, 1961) was able to obtain the first useful spectrum of the zodiacal light in 1959 at the Chacaltaya observatory (Alt. 5,400 m) of the Universidad Mayor de San Andres, La Paz, Bolivia. This spectrum was the result of several hours exposure spread over seven nights of observation, and it

Copy right $(\subset)$ The Society of Geomagnetism and Earth, Planetary and Space Sciences (SGEPSS); The Seismological Society of Japan; The Volcanological Society of Japan; The Geodetic Society of Japan; The Japanese Society for Planetary Sciences. showed for the first time that the zodiacal light is sunlight scattered from particles comparable in size with the wavelength of light, and not, as was thought possible at the time, from free electrons in interplanetary space.

Interest then broadened to include the possibility of observing a Doppler shift in the scattered light, as might be expected if the scattering particles were in planetary orbits around the sun. Various workers, such as Ingham (Ingham, 1963), and the present author (James, 1969) made theoretical investigations of the changes that might be expected to the profiles of Fraunhofer lines by the scattering process, and optical design studies showed that the possibility existed of measuring the line shapes.

\section{Photon-Counting Monochromators}

In the early 1960's the technique of photon-counting in the visible region had developed to the state where a photoncounter could be employed as the detector in a monochromator. This represented the state of the art at the time. The monochromator is very inefficient since it discards most of the received signal, and works only because the quantum efficiency of a photomultiplier photocathode, about 0.2 , is so much greater than that of a grain of photographic emulsion, which must receive about two thousand photons before it becomes developable. This high quantum efficiency more than compensates for the fact that all the wavelength channels are open simultaneously in a spectrograph. There are other, more subtle advantages too. In a monochromator the numerical aperture is unimportant: all that is required is that the photons which pass through the pupil of the system arrive eventually at the detector. In other words, the photons arriving per unit area is not a criterion as it is with a photographic plate and this makes the optical design much easier. Aberrations are reduced simply by using a large focal ratio.

However the advantage of this system begins to disappear at very low light levels where the random noise from the refrigerated green-sensitive photomultiplier cathode, equivalent to about 10 photons s $^{-1}$ ( 2 photoelectrons s ${ }^{-1}$, and augmented on Chacaltaya by the cosmic ray flux of about 7 events $\mathrm{s}^{-1}$ ) begins to ruin the statistics of the photon counting. This is what happened to the first attempt at photoelectric 


\section{Ingham's Chacaltaya Spectrograph, 1959.}

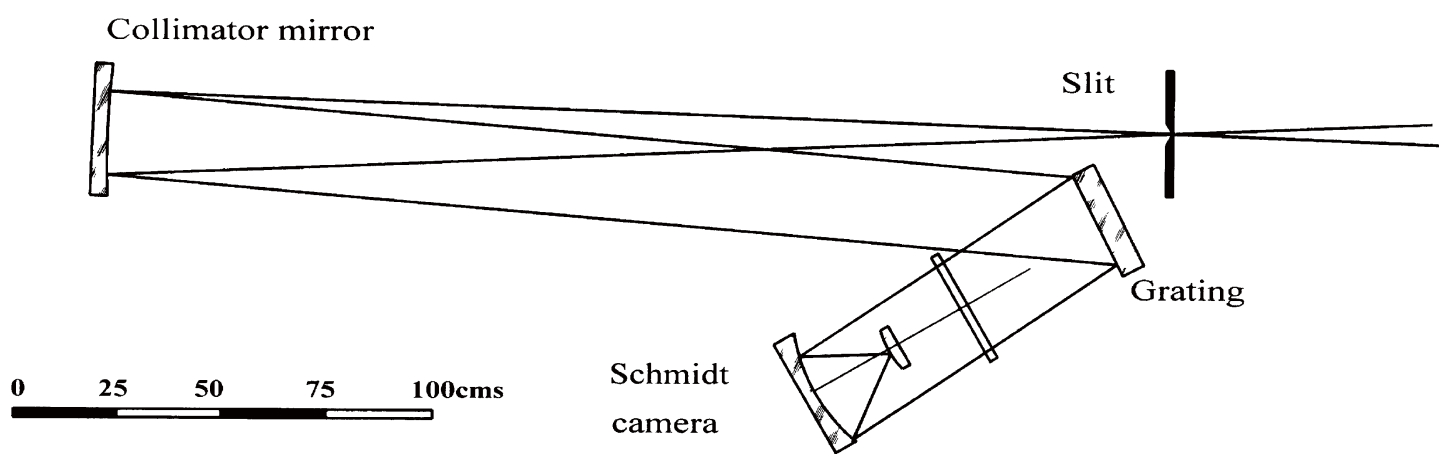

Fig. 1. This represents the ultimate in silver-halide spectrography, where the low sensitivity of the photographic emulsion is compensated by the very high numerical aperture achievable by the Schmidt optical system. The position of the photographic plate inside the camera shows why it is impossible to contain the bulk of a refrigerated CCD chip in a Schmidt system.

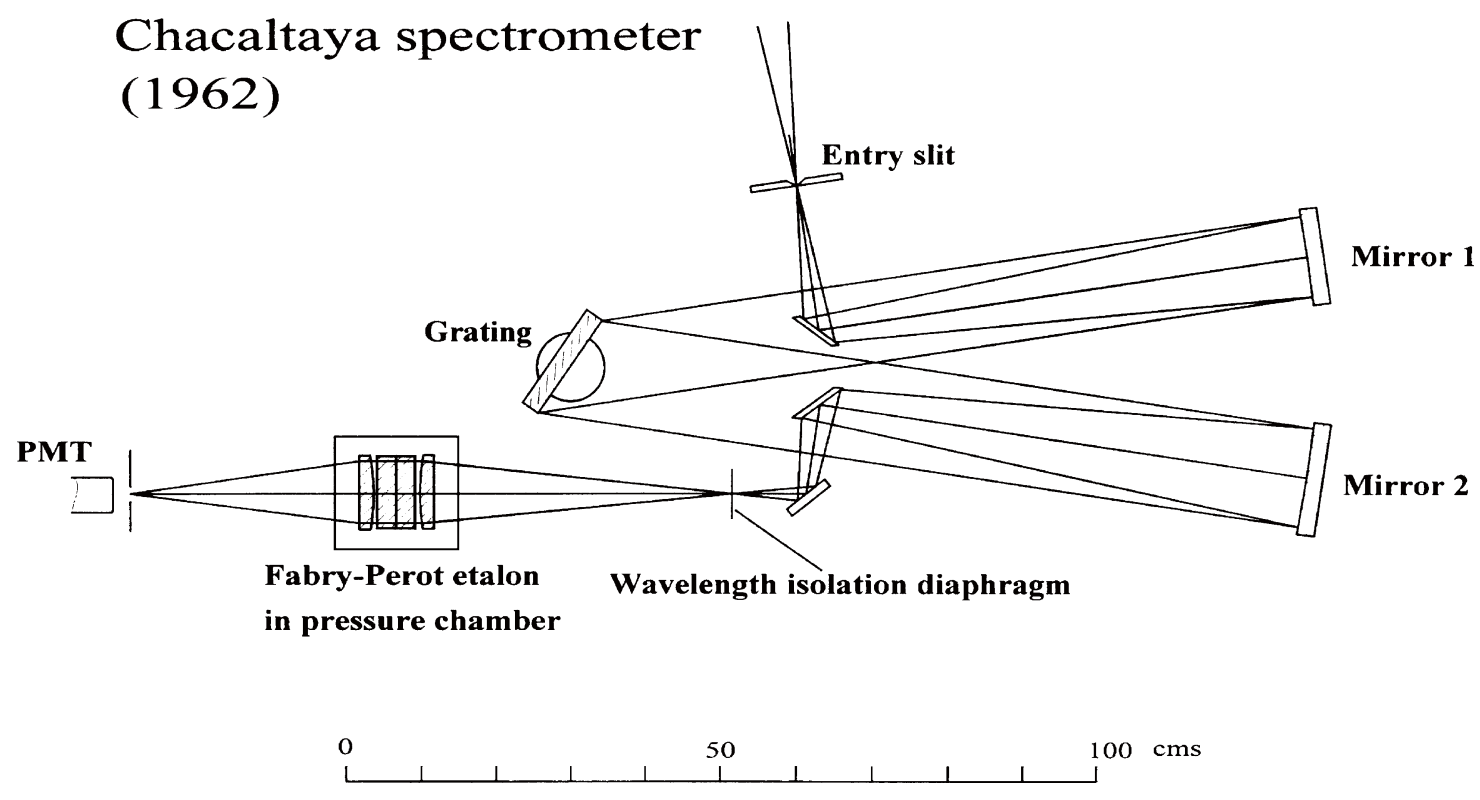

Fig. 2. This is the first attempt at interference spectrometry, before the advent of stable interference filters. The spectrograph acts as the broad-band wavelength filter to isolate one free spectral range of the Fabry-Perot étalon. The wavelength scanning is done by variation of the gas pressure inside the étalon pressure-chamber.

spectrometry of the zodiacal light. The light flux obtained at $1.2 \AA$ resolution was of the order of $8-10$ counts $\mathrm{s}^{-1}$ and an inordinate time was needed to get a reasonable and useful signal/noise ratio.

The first zodiacal light spectrometer employed a CzernyTurner spectrograph as a pre-filter and a Fabry-Perot étalon as the main disperser. The étalon was scanned in wavelength by varying the pressure of gas between the plates. A Freontype gas was used because of its high refractive index and a scan through one order of the étalon was possible in about ten minutes. However, there was a further complication introduced by the fact that the Chacaltaya observatory is at $5,400 \mathrm{~m}$ where the ambient temperature is not only below the freezing point of water but below the boiling point of freon; and liquid freon in the étalon chamber was a distinct embarrassment.

Although two attempts were made to do spectrometry in this way it is probably safe to say that no significant result was obtained, but that there was a deeper understanding of the problems involved. One non-problem was also illuminated. It was thought that the Hydrogen $\mathrm{H} \beta$ line might be emission in the night sky (as $\mathrm{H} \alpha$ occasionally is), and that the choice of this Fraunhofer line was an unfortunate one. However, after several years of airglow spectrometry at high sensitivity, I am fully persuaded that this is not so.

The Czerny-Turner spectrometer was employed because at the time the art of interference filter construction was in its infancy and no adequate filters were yet available.

By 1969 the situation had changed in two respects:

(1) Servo-controlled and stabilised Fabry-Perot étalons were feasible (Ramsay, 1962; Smeethe and James, 1971).

(2) Interference filters of adequate stability and aperture were available.

So a new type of monochromator was designed to take 
Haleakala, 1969

Fabry-Perot Spectrograph.

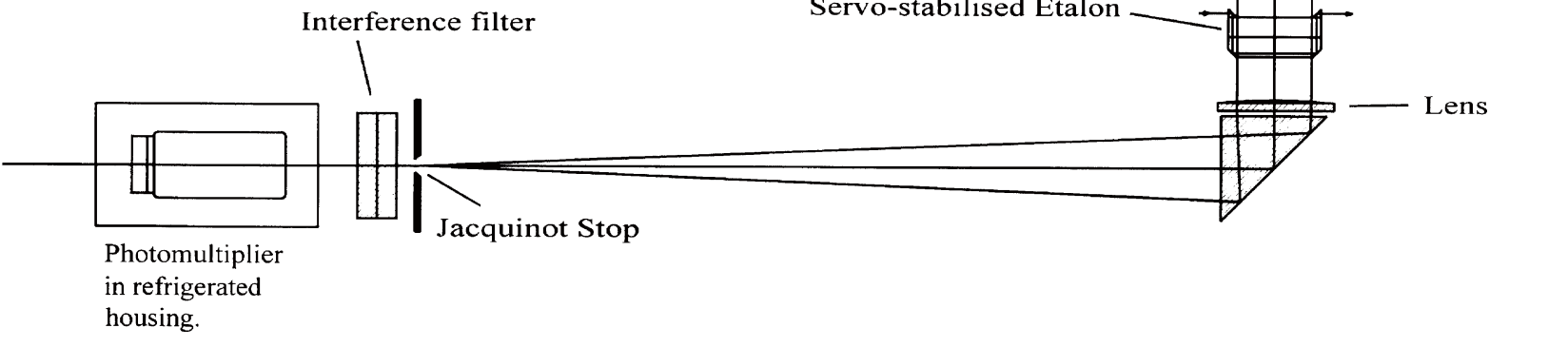

$\begin{array}{lllll}0 & 100 & 200 & 300 & 400\end{array} 500 \mathrm{~mm}$

Fig. 3. The optical system becomes much simpler when an interference filter is used. In this case the wavelength scanning is done by mounting the étalon plates on piezo-electric transducers and applying a sawtooth waveform. A separate optical servo-system holds the gap parallel during the scanning.

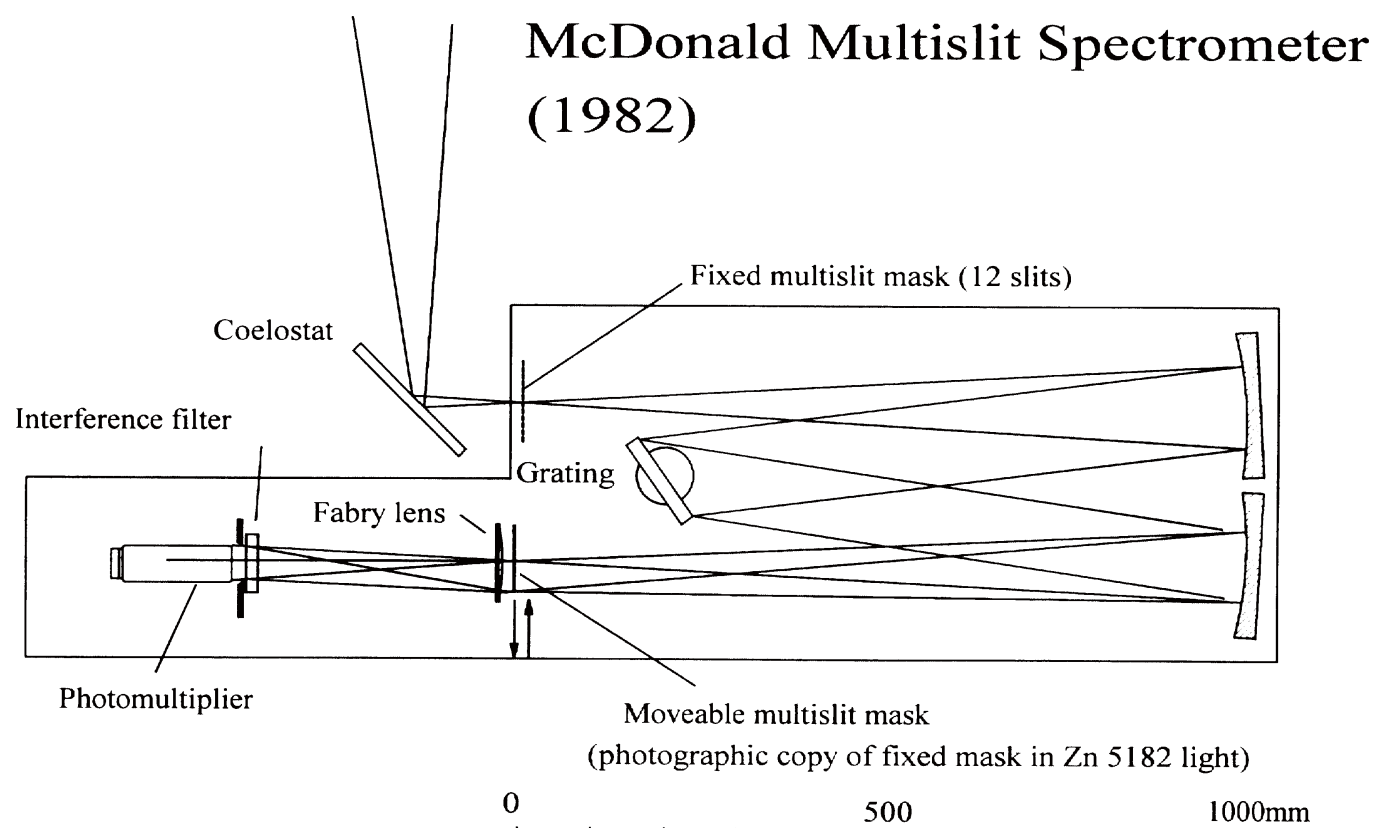

Fig. 4. This is a variation on the Griffin-system used by Fried. There are multiple entry slits and an interference filter isolates a $20 \AA$-wide wavelength band, so that images of the entry slit appear separated at the focal plane. Scanning is done by moving a mask transversely across the focal plane and the grating is imaged on to the photomultiplier cathode by a Fabry-lens just beyond the focal plane.

advantage of these developments. This was first used in 1969 (James and Smeethe, 1970) at the Haleakala observatory of the University of Hawaii (Alt. 3,000 m), and Doppler shifts more or less agreeing with expectation and theory were measured. The design was much simplified, and the photomultiplier was cooled with commerically available dry-ice, instead of the home-made product which was required at Chacaltaya. Consequently the time available for observation was much increased as was the photon flux. A signal/noise ratio of 20:1 was available in a fifteen minute exposure, during which a scan through a range of $10 \AA$ was made.

Dr. Reay's group (East and Reay, 1984) made similar measurements at the Izaña observatory in Tenerife (Alt. 2,440 m), with similar results.

An alternative approach was by Fried (1978), who used a Griffin-style radial velocity spectrometer (Griffin, 1967) with a cooled photomultiplier. The principle is that a spec- 


\section{Haleakala Spectrograph (1995)}

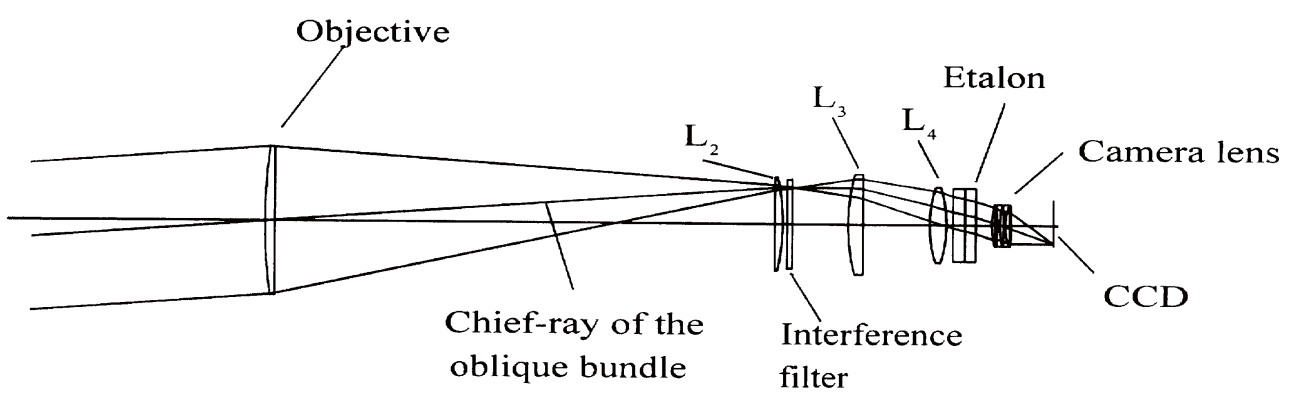

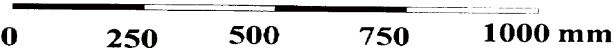

Fig. 5. This represents an improvement of several orders of magnitude over previous instruments. The high sensitivity of the CCD chip is combined with high numerical aperture optics to obtain photographs of complete Fabry-Perot rings. As before, the spectral region of interest is isolated with an interference filter and the sky is imaged on to the CCD chip along with two or three orders of Fabry-Perot rings. Instrusive star images can be blotted out by suitable programming, they being generally too bright to be considered when subsequently analysing the frame in the computer.

trograph has its focal plane covered by a plate with a negative photographic image of the solar spectrum, to that an un-shifted spectrum from any source of scattered sunlight is not transmitted: the plate is blackened wherever there is light. But is there is a Doppler shift it is necessary to move the plate to obtain extinction and the movement of the plate measures the shift. It is only approximate: the Doppler effect stretches the spectrum rather than moving it (each wavelength is multiplied by a factor very slightly different from unity), so that only a limited range of spectrum may be employed. This method, when designed to the practical limit, has a detective quantum efficiency similar to that of the Fabry-Perot étalon spectrometer and has the advantages of being somewhat easier to construct and more robust to use in rough conditions.

The disadvantage is that deep Fraunhofer lines are sparse in the solar spectrum and most of the spectral range does not contribute to the observation. A variant of this principle was constructed by the author, who, instead of using a length of spectrum, used an interference filter and a multiple input slit. The exit slit was a photograph of the input slit in the light of the zinc line at $5182 \AA$, this arrangement being made necessary by various non-linearites in the optics (equally spaced straight entry slits give curved images with unequal separations). It was used at the McDonald observatory of the University of Texas but the expedition was frustrated by the eruption of a volcano in Mexico which produced a zenith extinction of 3.5 magnitudes (a factor of 40).

\section{CCD Spectrography}

There the matter appears to have rested for some years, the currently available technology having been taken to its limits. However, in the 1980's the cooled charge coupled device became reliable and cameras became commercially available in a portable form. The CCD is in essence a photographic plate with the quantum efficiency of a photomultiplier - somewhat better in fact, a Detective Quantum Efficiency (DQE) about $0.35 \rightarrow 0.5$ as compared with the
$0.2 \rightarrow 0.3$ from photocathodes, or in photographic terms, an ISO rating of 2 or 3 million. The author then considered the possibility of combining this device with a Fabry-Perot étalon to produce a complete ring-system on a CCD chip at the highest possible numerical aperture (James, 1996). As mentioned earlier, with a photographic system as opposed to a photon-counting system, it is the relative aperture that matters, the "focal ratio" or the numerical aperture. The bulk of the CCD camera prevented the use of Schmidt optics in the Ingham fashion, and so an optical design study was done to see whether an F/1.4 beam could be brought to a focus on a CCD which would give sufficient resolution to allow ring diameters to be measured with the required accuracy.

The answer was that it was possible, and that a $15 \mathrm{~cm}$ aperture telescope would image a patch of sky $3^{\circ}$ diameter on the CCD ship with 2.5 orders of interference from centre to edge. The essence of the design is that the camera pupil and not the étalon must be the pupil of the system, and the étalon must be slightly larger than the pupil diameter.

There was another problem to be faced: that of interpreting the data. The Fabry-Perot rings are an awkward shape and the dispersion is non-linear, so a computer programme was required to examine the contents of each pixel of the CCD image and sort the contents into a wavelength-linear array. The étalon gap was chosen to give a resolution of $1 \AA$, and a spectral range of $29 \AA$ emerged in practice. For calibration, a vapour discharge-lamp, filtered to transmit the $\mathrm{Zn}$ line at $5182 \AA$ was used, and the Fraunhofer lines of the Mg triplet as $\lambda \lambda 5167,5172,5184 \AA$ in the solar spectrum were used as the wavelength range to be observed. The spectral range was isolated with an interference filter of $12 \AA$ FWHM and passband centered on $5180 \AA$.

In practice it was found possible to obtain ring patterns everywhere in the sky. They were more intense obviously in the ecliptic plane, but vestigial (although not useable) rings could be seen, even at high ecliptic latitudes and elongations. It is impossible to say whether this light is scattered from interplanetary dust or whether perhaps it is contributed by 
the diffuse stellar background from predominantly G-type stars.

A cursory look at the images show that there is a considerable improvement in performance over previous spectrometers and that useful images can be obtained at elongations out to $90^{\circ}$ elongation in an exposure time of $900 \mathrm{~s}$.

\section{Future Developments}

Camera lenses with high relative aperture $(\mathrm{F} / 1.4 \rightarrow \mathrm{F} / 1.2)$ are commerically available but their resolution is not yet adequate for resolution of the outer rings of the Fabry-Perot pattern. CCD's with larger areas are becoming available, and this means that longer focal length camera lenses can be used with larger étalons. The number of photons arriving per second at each pixel is unchanged, but there are more pixels and hence better statistics in the analysis. The telescope focal length can be increased, covering a smaller area on the sky and giving consequently a smaller spread of elongation.

In the end it is the total area of the CCD that controls the efficiency of the instrument. The size of the pixels is unimportants so long as the rings are adequately resolved at the edge: indeed, there is an advantage in having large pixels rather than small ones, since the read-out noise is correspondingly less.

There is now (1997) needed a search for an adequate observing site. Most astronomical observatories, while adequate for stellar and cosmological observations, are not sufficiently dark and unpolluted for these delicate measurements of zodiacal light and gegenschein. The problem is to find somewhere safe from political turmoil, near the equator, at high altitude, with reasonable weather and cloudcover in March and September* and with liquid nitrogen within reach $* *$ (Peltier-cooled CCD cameras are not suitable for the long exposures needed).

\section{References}

Blackwell, D. E. and M. F. Ingham, Observations of the zodiacal light from a very high altitude station, Mon. Not. Royal Astron. Soc., 122, 129141, 1961.

East, I. R. and N. K. Reay, The motions of interplanetary dust particles, Astron. Astrophys., 139, 512-516, 1984.

Fried, J. W., Doppler shifts in the zodiacal light spectrum, Astron. Astrophys., 68, 259-264, 1978.

Griffin, R. F., A photoelectric radial velocity spectrometer, Astrophys. J., 148, 465-476, 1967.

Ingham, M. F., The profile of an absorption line in the spectrum of the zodiacal light, Mon. Not. Royal Astron. Soc., 126, 377-380, 1963.

James, J. F., Theoretical Fraunhofer line profiles in the spectrum of the zodiacal light, Mon. Not. Royal Astron. Soc., 142, 45-52, 1969.

James, J. F., A radial velocity spectrograph for the zodiacal light, Mon. Not. Royal Astron. Soc., 280, 1055-1061, 1996.

James, J. F. and M. J. Smeethe, Motion of the interplanetary dust cloud, Nature, 227, 588-589, 1970.

Ramsay, J. V., A rapid-scanning Fabry-Perot interferometer with automatic parallelism control, Appl. Optics, 1, 411-413, 1962.

Smeethe, M. J. and J. F. James, A servo-stabilised Fabry-Perot spectrometer, J. Phys. E, 4, 429-432, 1971.

\footnotetext{
*When the Milky Way causes least interference.

**Computers work without problem from portable petrol-powered generators.
} 